\title{
Towards 5G millimeter-wave wireless networks: a comparative study on electro-optical upconversion techniques
}

\author{
Nael A. Al-Shareefi ${ }^{1}$, Jaafar A. Aldhaibaini' ${ }^{2}$, Sura Adil Abbas ${ }^{3}$, Hadeel S. Obaid ${ }^{4}$ \\ ${ }^{1}$ College of Biomedical Informatics, University of Information Technology and Communications (UOITC), Iraq \\ ${ }^{2,3,4}$ College of Engineering, University of Information Technology and Communications (UOITC), Iraq \\ ${ }^{1}$ Dijlah University College, Baghdad, Iraq
}

\begin{tabular}{l} 
Article Info \\
\hline Article history: \\
Received Oct 2, 2019 \\
Revised Feb 9, 2020 \\
Accepted Mar 29, 2020 \\
\hline Keywords: \\
Comparative study \\
Eye pattern \\
Fifth-generation (5G) \\
Q-factor \\
Tupling-techniques
\end{tabular}

Tupling-techniques

\begin{abstract}
Fifth-generation $(5 \mathrm{G})$ wireless networks that use the $\mathrm{MM}-\mathrm{W}$ hold a great promise to revolutionize wireless industry. However, the difficulty in generating and transmitting these high-frequency signals in the electricaldomain due to bandwidth limitation of electronic components, and high absorption loss limits current applications. Consequently, ptical generation and transmission of $\mathrm{MM}-\mathrm{W}$ signals are a viable option. In this paper, a comparative study is carried out on three electro-optical upconversion (EOU) techniques to generate $60-\mathrm{GHz} \mathrm{MM}-\mathrm{W}$ signal, namely 4-tupling, 6-tupling and 8-tupling. The paper briefly describes the three techniques and analyses the optical harmonic distortion suppression ratio (OHDSR) and electrical spurious suppression ratio (ESSR) generated by each one of the techniques. OHDSR and ESSR have been compared to show the trade-off between the techniques. In addition, the paper compares the implementation of non-ideal phase shifting on OHDSR for the three EOU techniques. Finally, the performance of the three EOU techniques after transmission over optical fiber is evaluated by quality factor (Q-factor) and eye pattern test. The results of the simulation illustrate well the benefits of the performed study and confirm that the 4-tupling constitutes a cost-effective technique to generate MM-W signals.
\end{abstract}

Copyright $\odot 2020$ Institute of Advanced Engineering and Science. All rights reserved.

\section{Corresponding Author:}

Nael A. Al-Shareefi,

College of Biomedical Informatics,

University of Information Technology and Communications (UOITC), Baghdad, Iraq.

Email: dr.nael.al_shareefi@uoitc.edu.iq

\section{INTRODUCTION}

Wireless industry is moving toward the 5G NR (New Radio) technology to provide unprecedented 7 Gigabit-per-second throughput to a mobile device. One way to enable $5 \mathrm{G}$ is to click on unused new bands at the top of the radio spectrum. These high bands are known as MM-W and have been recently been opened up by regulators for licensing [1]. MM-W electrical generation and transmission look troublesome process since $\mathrm{MM}-\mathrm{W}$ signals are susceptible to interference and usually need to maintain line-of-sight transmission to work [2]. Besides, MM-W signals cannot easily move through buildings or obstacles and they can be absorbed by leaves and rain [3]. Hence optical $\mathrm{MM}-\mathrm{W}$ generation and $\mathrm{R}-\mathrm{O}-\mathrm{F}$ transportation techniques were proposed. The optical $\mathrm{MM}-\mathrm{W}$ generation techniques can be broken down into optical heterodyne detection (OHD) [4-7] and EOU techniques [8-14]. EOU gained a lot of attention because of its stability, flexibility and higher modulation bandwidth. EOU is a technique based optical frequency-multiplication wherein high-order optical harmonics are created by using a laser diode with an external modulator, such as Mach-Zehnder modulator (MZM) driven by a sinusoidal radio frequency (RF) signal. Compared with 
the OHD technique, EOU can generate a high-purity optical MM-W signals without the use of complex mechanisms for phase noise repression [15].

Several different EOU techniques have been recently proposed with various frequency-multiplication such as 4-, 6-, 8-, 12-, 16- and 24-tupling. Author [16] proposes a frequency 24-tupling MM-W generation technique based on two integrated MZM (IMZMs). Authors [17, 18] propose 16-tupling technique using two cascaded MZMs and dual parallel MZMs. Author [19] has proposed 12-tupling technique. This technique is based on based on two cascaded dual-parallel MZMs. MM-W generation with higher frequency multiplication factors, i.e. 24,16 and 12 , plays a significant role in reducing requirements for high-frequency RF LOs at the switching center. However, complex circuits and higher modulation index are needed. The higher modulation index will has a low conversion efficiency. Furthermore, ESSR and OHDSR are low [20]. The present paper is focusing on EOU techniques for optical generation and transmission of $\mathrm{MM}-\mathrm{W}$ signals. A comparative study is carried out between three different EOU techniques. The study aims at showing strengths and weaknesses of each EOU technique and evaluating each technique in terms of OHDSR and ESSR. As well as in terms of the influence of non-ideal phase shifting. The organization of this paper is as follows; Section 2 gives a brief description of the three selected EOU techniques. Section 3 details the simulation results and discussions. Subsequently, Section 4 concludes the paper.

\section{TUPLING-TECHNIQUES}

\subsection{4-Tupling technique}

This frequency-multiplication technique is based on two parallel dual-drive MZMs to generate high-quality optical carrier suppression (OCS) $\mathrm{MM}-\mathrm{W}$ signal. The OCS based frequency-multiplication improve $\mathrm{R}-\mathrm{O}-\mathrm{F}$ transmission systems due to its dispersion tolerance [21]. Figure 1 shows the schematic diagram for this technique [22].

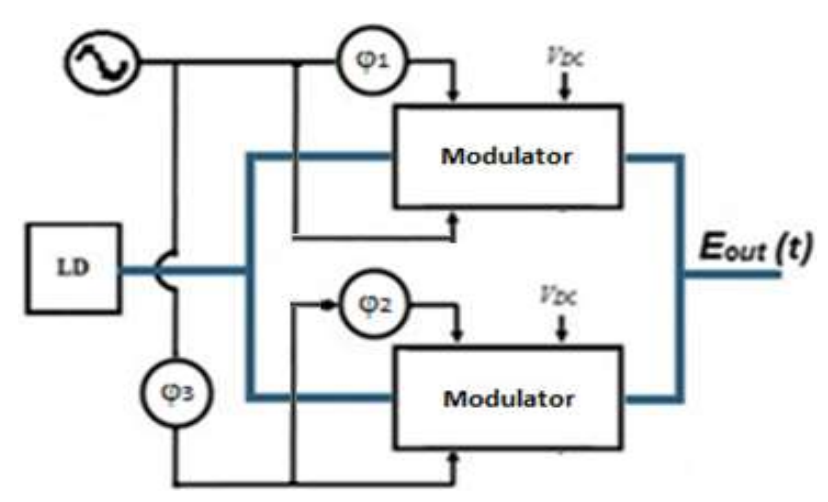

Figure 1. Schematic diagram of the 4-tupling technique [22]

The generated optical signal can be written as [22]

$$
E(t)=2 J_{2}(m) \cdot e^{i\left(\omega_{c}+2 \omega_{R F}\right)}
$$

Only $2^{\text {nd }}$-order optical carriers are obtained. where $\omega_{c}, \omega_{R F}$ are the frequency of the optical signal and electrical driving signal and $J_{2}$ is the first kind Bessel function of the $2^{\text {nd }}$-order. OHDSR is the power-level difference between the main optical carrier and unwanted optical harmonics. The equation of OHDSR can be derived using (1):

Harmonic suppression $=10 \log _{10}[(\text { Numerator/Denominator })]^{2}$

Simplifying the numerator of (2), yield

$=2 J_{2}^{2}$

Simplifying the denominator of (2), yield 
$=2 J^{2}$

\section{Harmonic suppression=42.01 dB}

ESSR is the power-level difference between the required electrical MM-W signal and spurious $\mathrm{MM}-\mathrm{W}$ signal. The equation of ESSR for this technique is given by:

\section{Harmonic suppression=20 $\log _{10}[($ Numerator/Denominator $)]$}

Simplifying the numerator of (4), yield

$=4 J_{6} J_{2}+2 J_{2}^{2}$

Simplifying the denominator of (4), yield

$=4 J_{6} \cdot J_{2}$

\section{Harmonic suppression $=36.1 \mathrm{~dB}$}

By using this technique, an OHDSR more than $42 \mathrm{~dB}$ and an ESSR more than $36 \mathrm{~dB}$ are attained at a modulation index $\mathrm{m}=2.404$.

\subsection{6-Tupling technique}

A 6-tupling technique that can generate a $60-\mathrm{GHz}$ MM-W signal using an IMZMs was developed by Shi et al. Figure 2 shows the schematic diagram for this technique [23]. The technique uses three sub-MZMs. The electrical phase shift between the two RF drive signals of the two sub-MZMs is $\varphi=144^{\circ}$.

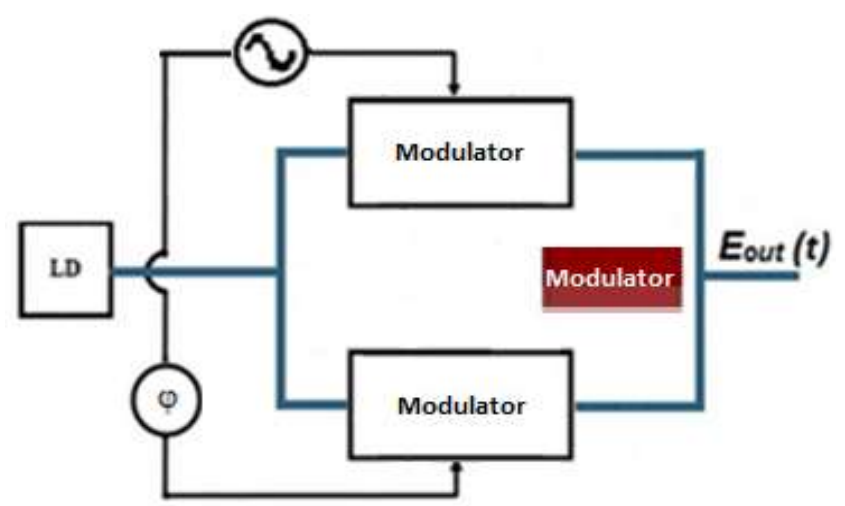

Figure 2. Schematic diagram of the 6-tupling technique [23]

The generated optical signal can be written as [23]

$$
E(t)=2 J_{3}(m) \cdot e^{i\left(\omega_{c}+3 \omega_{R F}\right)}
$$
using (6):

Only $3^{\text {rd }}$-order optical carriers are obtained. The equation for harmonic suppression can be derived

Harmonic suppression $=10 \log _{10}[(\text { Numerator/Denominator })]^{2}$

Simplifying the numerator of (7), yield [23]

$=J_{3}(m)$

Simplifying the denominator of (7), yield [23]

$=J_{7}(m)$ 


\section{Harmonic suppression $=31 \mathrm{~dB}$}

ESSR is the power-level difference between the required electrical signal and spurious signal. The equation of ESSR for this technique is given by

\section{Harmonic suppression=20 $\log _{10}[($ Numerator/Denominator $)]$}

Simplifying the numerator of (9), yield [23]

$=J_{3}(m)$

Simplifying the denominator of (9), yield [23]

$=2 J_{7}(m)$

\section{Harmonic suppression $=25 \mathrm{~dB}$}

Utilizing this technique, an OHDSR more than $30 \mathrm{~dB}$ and an ESSR more than $25 \mathrm{~dB}$ are obtained. A modulation index $(\mathrm{m}=3.831)$ is required to drive the IMZM.

\subsection{Eight-tupling technique}

An 8-tupling technique that can generate a MM-W signal using four nested MZMs was developed by Shang etal. Figure 3 shows the schematic diagram for this technique [24]. The technique comprise of two 4-tupling systems. Each system is consisting of two cascaded MZMs with a $90^{\circ}$ phase delay between RF signals. The generated optical signal is written as [24]

$$
E(t)=2 J_{4}(m) \cdot e^{i\left(\omega_{c}+4 \omega_{R F}\right)}
$$

Only $4^{\text {th }}$-order optical carriers are obtained. The technique offers a high-quality optical and electrical generation, Optical harmonic suppression $=44 \mathrm{~dB}$ and $\mathrm{RF}$ harmonic suppression=44 $\mathrm{dB}$, at $\mathrm{m}=3.831$. In addition, the system is not affected by the biased deviation for MZM, indicating a higher stability. Nevertheless, higher modulation index and complexity are the essential restrictions.

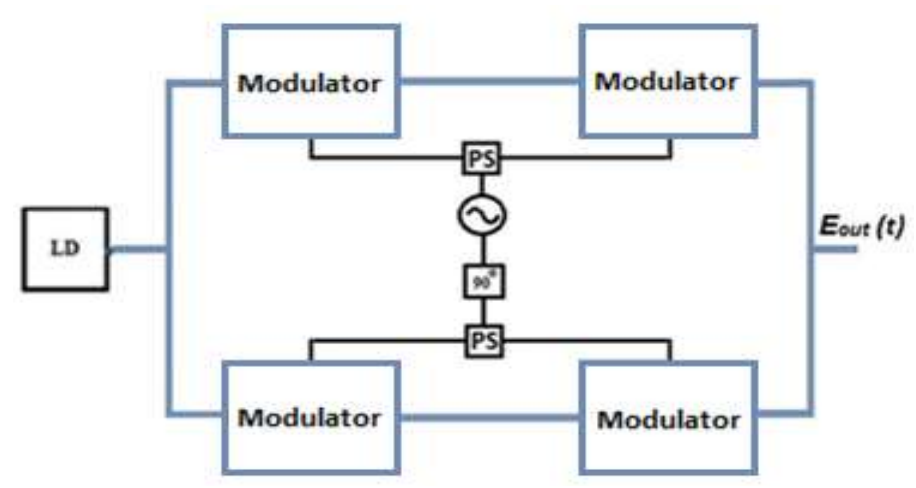

Figure 3. Schematic diagram of the 8-tupling technique [24]

\section{SIMULATION RESULTS AND ANALYSIS}

The simulation system for B-T-B is set up for the three techniques by using "OptiSimTM 9.0", to evaluate the values of OHDSR and ESSR. Figure 4 shows the simulated experimental setup for the 4-tupling technique, i.e. Figure 1.

To evaluate the effect of the variation of phase shifting on harmonics-suppression for the three EOU techniques, the phase shift (PS) is varied from $-9^{\circ}$ to $9^{\circ}$, and its effect on harmonics-suppression is shown in Figure 5. For 4-tupling and 8-tupling techniques, the power ratio (OHDSR) is changed. The highest harmonics-suppression values occur at phase shifts near zero. Then the values decrease with increasing the phase shift deviation as shown in Figure 8. An harmonics-suppression of $25 \mathrm{~dB}$ and $21 \mathrm{~dB}$ can 
be attained if the deviation is $9^{\circ}$. For 6 -tupling technique, harmonics-suppression is more sensitive to phase difference deviation. However, for the three EOU techniques, considerable harmonics-suppression degradation occurs with phase shifting more $4^{\mathrm{O}}$ or $-4^{\mathrm{O}}$.

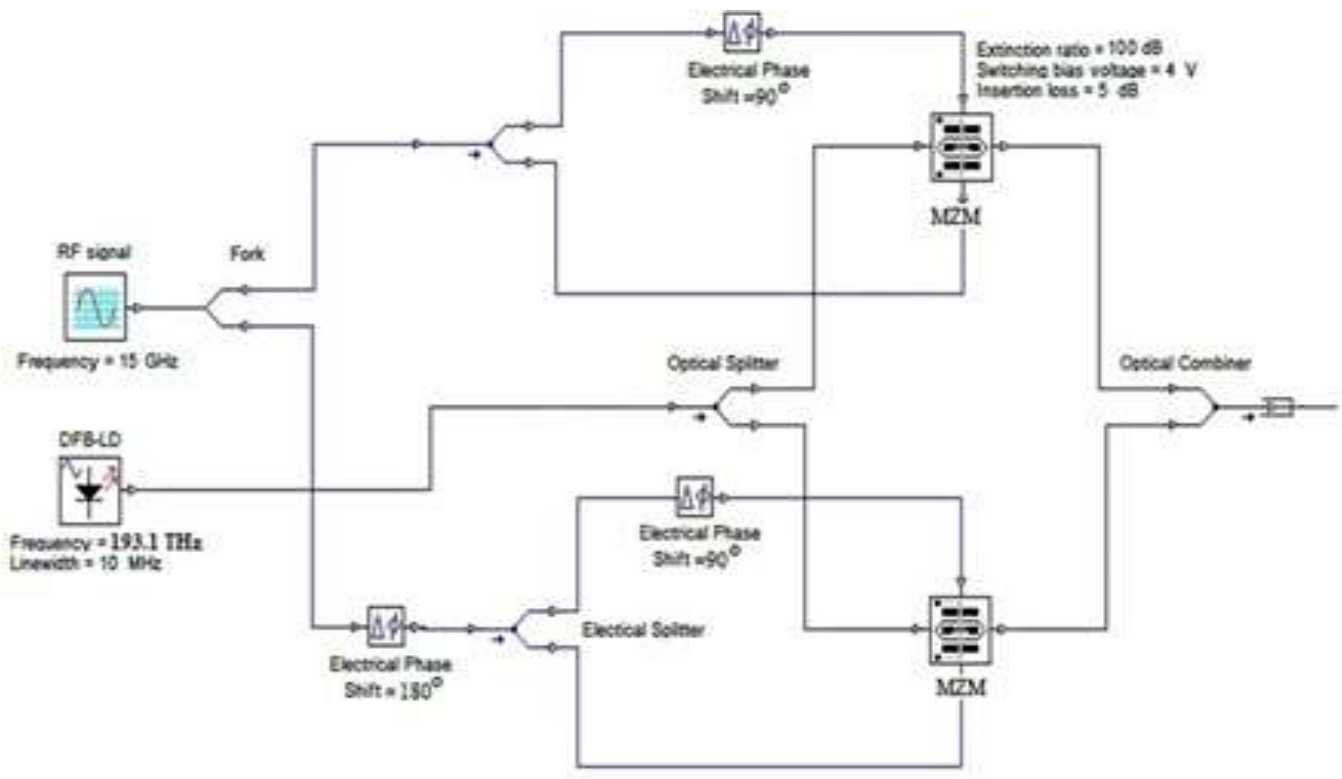

Figure 4. Simulated Experimental setup of the 4-tupling MM-W generation technique

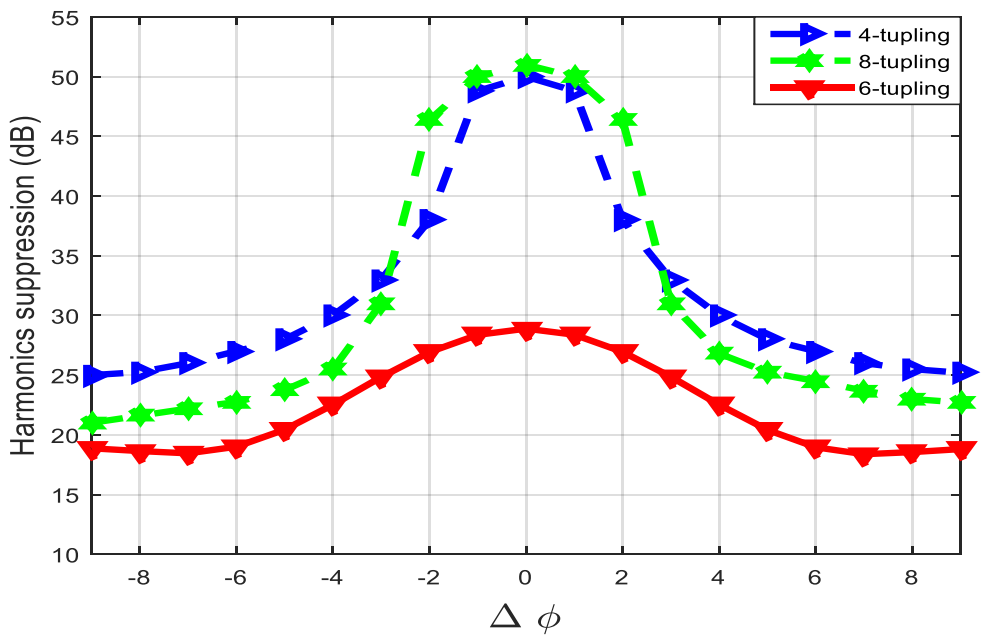

Figure 5. Harmonics suppression Vs $\Delta \phi$

To further evaluate the performance of each EOU technique studied, simulated electrical eye patterns are shown in Figure 6 for a transmission distance of $60 \mathrm{~km}$. Attenuation, chromatic dispersion and nonlinear effect are all activated in accordance with industry standards. Figure 6a shows the simulated electrical eye pattern of the recovered baseband signal for the 4-tupling technique. The eye pattern outline remains open and clear although the optical $\mathrm{MM}-\mathrm{W}$ signal is transmitted over $60 \mathrm{~km}$. The $\mathrm{Q}-$ factor is approximately 5.8 as depicted in Figure 7 a.

Figure $6 \mathrm{~b}$ shows the simulated electrical eye pattern for the 6 -tupling technique. The eye pattern retains open and the pulse width of the demodulated data becomes broader. The pulse width becomes broader and broader with increasing fiber length and that the bit walk-off effect gradually becomes serious, especially at $65 \mathrm{~km}$. The Q-factor is approximately 5.2 as depicted in Figure 7b. Figure 6c shows the simulated electrical eye pattern for the 8-tupling technique. The eye pattern closes and the performance becomes unacceptable at a distance of $60 \mathrm{~km}$. The Q-factor is approximately 3.8 as depicted in Figure 7c.

Towards 5 G millimeter-wave wireless networks: a comparative study on... (Nael A. Al-Shareefi) 
The physical cause is the effect of bit walk-off [25]. As seen from Figures 6, recovered baseband signal for 6- and 8-tupling techniques suffer from oscillations in RF power bigger than 4-tupling technique due to fiber nonlinear distortion and bit walk-off effect.

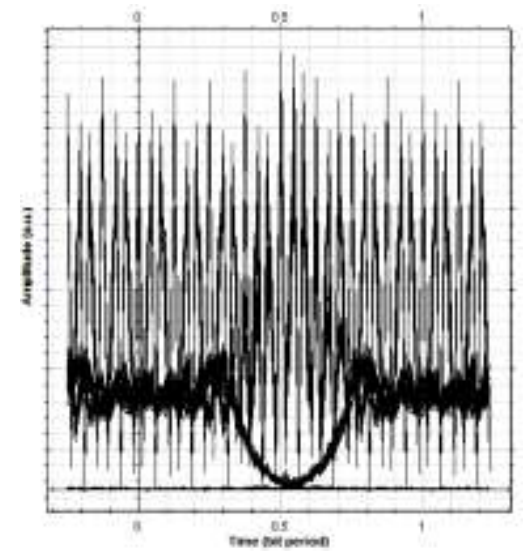

(a)

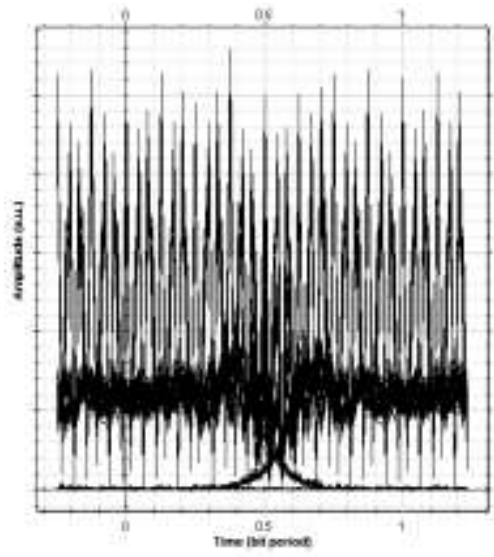

(b)

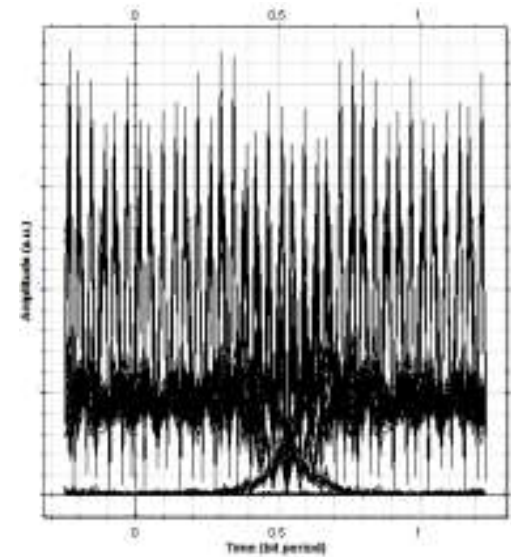

(c)

Figure 6. Simulated eye patterns at transmission distances of $60 \mathrm{~km}$, (a) 4-tupling technique,

(b) 6-tupling technique, (c) 8-tupling technique

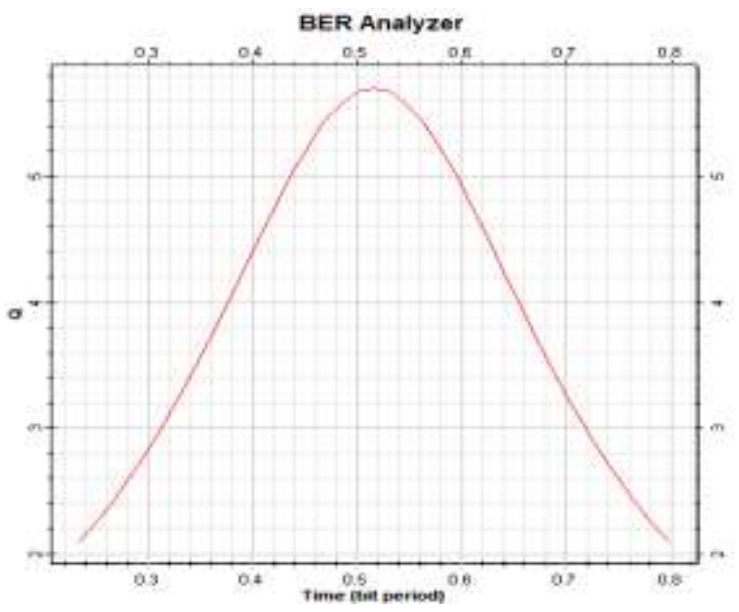

(a)

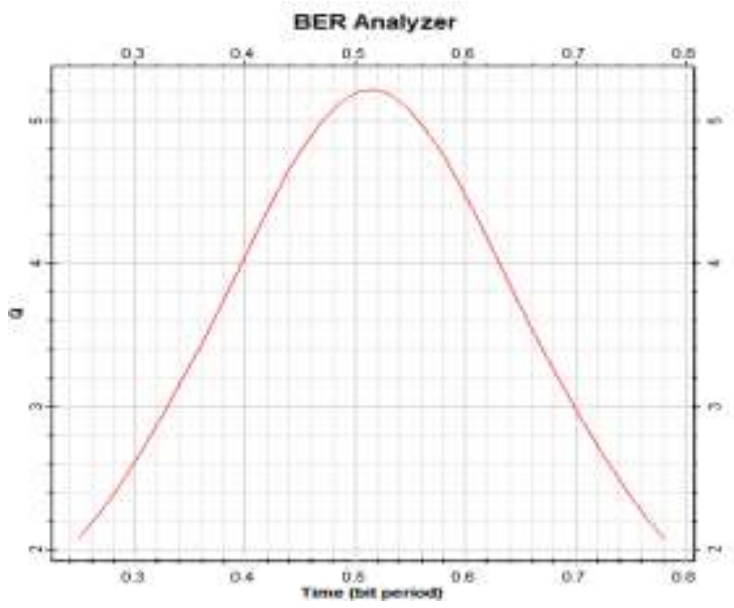

(b)

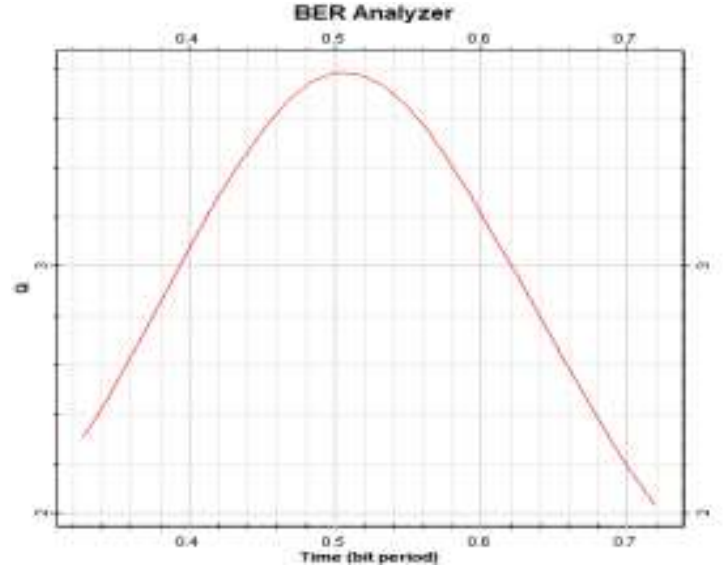

(c)

Figure 7. Simulated Q-factor at transmission distances of $60 \mathrm{~km}$, (a) 4-tupling technique, (b) 6-tupling technique, (c) 8-tupling technique 


\section{CONCLUSION}

This comparative study confirms that the 4-tupling constitutes a cost-effective technique to generate 60-GHz MM-W signal. By comparing OHDSR, ESSR, eye patterns and Q-factor values of three different EOU techniques, we realized that the 4-tupling technique can generate a stable MM-W signal with a lower modulation index than $6-$ and -8 tupling techniques. Moreover, MM-W signal can resist the fading caused by the variation of phase shifting. However, the 4-tupling technique is not adequate to minimize requirements on the electrical components for wireless applications at frequencies above 100-GHz. Frequency -6 and -8 tupling techniques can minimize the requirements significantly. Unfortunately, Frequency -6 and -8 tupling techniques techniques present two disadvantages: first, they require a higher modulation index. Second, MM-W signal suffer from oscillations in RF power due to fiber nonlinear distortion and bit walk-off effect.

\section{REFERENCES}

[1] U. Farooq and G. M. Rather, "Millimeter wave (MMW) communications for fifth generation (5G) mobile networks," in Progress in Advanced Computing and Intelligent Engineering, ed: Springer, pp. 97-106, 2019.

[2] N. D. Anh and D.-T. Do, "The Maximal SINR Selection Mode for 5G Millimeter-Wave MIMO: Model Systems and Analysis," Indonesian Journal of Electrical Engineering and Computer Science, vol. 7, no. 1, pp. 150-157, 2017.

[3] A. A. Budalal, I. M. Rafiqul, M. H. Habaebi, and T. A. Rahman, "The effects of rain fade on millimetre wave channel in tropical climate," Bulletin of Electrical Engineering and Informatics, vol. 8, no. 2, pp. 653-664, 2019.

[4] K.-i. Kitayama, T. Kuri, H. Yokoyama, and M. Okuno, "60 GHz millimeter-wave generation and transport using stabilized mode-locked laser diode with optical frequency DEMUX switch," in Proceedings of GLOBECOM'96. 1996 IEEE Global Telecommunications Conference, pp. 2162-2169, 1996.

[5] M. Husaini, M. R. C. Beson, M. Anuar, R. Endut, and S. A. Aljunid, "Optical Generation of $80 \mathrm{GHz}$ Downstream Data in Radio over Fiber System Based on Stimulated Brillouin Scattering," in MATEC Web of Conferences, p. 01020, 2017.

[6] N. Qasem, "Enhancing the Capacity of the Indoor $60 \mathrm{GHz}$ Band Via Modified Indoor Environments Using Ring Frequency Selective Surface Wallpapers and Path Loss Models," International Journal of Electrical and Computer Engineering, vol. 8, no. 5, pp. 3003-3020, 2018.

[7] M. Hyodo, S. Saito, and Y. Kasai, "Optical phase-locked loop with fibre lasers for low phase noise millimetre-wave signal generation," Electronics letters, vol. 45, pp. 878-880, 2009.

[8] N. A. Al-Shareefi, et al., "A cost-effective method for high-quality $60 \mathrm{GHz}$ optical millimeter wave signal generation based on frequency quadrupling," Progress In Electromagnetics Research, vol. 137, pp. 255-274, 2013.

[9] J. Ma, C. Yu, Z. Zhou, and J. Yu, "Optical mm-wave generation by using external modulator based on optical carrier suppression," Optics communications, vol. 268, no. 1, pp. 51-57, 2006.

[10] C. S. Park, C. G. Lee and C. Park, "Photonic Frequency Upconversion by SBS-Based Frequency Tripling," in Journal of Lightwave Technology, vol. 25, no. 7, pp. 1711-1718, July 2007. doi: 10.1109/JLT.2007.897749.

[11] N. A. Al-Shareefi, et al., "Optical Generation of $60 \mathrm{GHz}$ Downstream Data in Radio over Fiber Systems Based on Two Parallel Dual-Drive MZMs," Int. J. Eng. Tech., vol. 6, no. 2, pp. 579-587, 2014.

[12] Y. Zhao, X. Zheng, H. Wen, and H. Zhang, "Simplified optical millimeter-wave generation configuration by frequency quadrupling using two cascaded Mach-Zehnder modulators," Optics Letters, vol. 34, no. 21, pp. 3250-3252, 2009.

[13] X. Liu, Z. Liu, J. Li, and P. Yue, "Frequency quadrupling using an integrated Mach-Zehnder modulator with four arms," Optics Communications, vol. 284, no. 16-17, pp. 4052-4058, 2011.

[14] N. A. Al-Shareefi, et al., "A study in OCS millimeter-wave generation using two parallel DD-MZMs," in 2013 IEEE 11th Malaysia International Conference on Communications (MICC), pp. 418-421, 2013.

[15] K. Kojucharow et al., "Simultaneous electrooptical upconversion, remote oscillator generation, and air transmission of multiple optical WDM channels for a $60-\mathrm{GHz}$ high-capacity indoor system," in IEEE Transactions on Microwave Theory and Techniques, vol. 47, no. 12, pp. 2249-2256, Dec. 1999. doi: 10.1109/22.808967

[16] X. Chen, L. Xia, and D. Huang, "A filterless 24-tupling optical millimeter-wave generation and RoF distribution," Optik-International Journal for Light and Electron Optics, vol. 147, pp. 22-26, 2017.

[17] Z. Zhu, S. Zhao, X. Chu, and Y. Dong, "Optical generation of millimeter-wave signals via frequency 16-tupling without an optical filter," Optics Communications, vol. 354, pp. 40-47, 2015.

[18] X. Yin, A. Wen, Y. Chen, and T. Wang, "Studies in an optical millimeter-wave generation scheme via two parallel dual-parallel Mach-Zehnder modulators," Journal of Modern Optics, vol. 58, no. 8, pp. 665-673, 2011.

[19] Z. Zhu, et al., "Filterless frequency 12-tupling optical millimeter-wave generation using two cascaded dual-parallel Mach-Zehnder modulators," Applied optics, vol. 54, no. 32, pp. 9432-9440, 2015.

[20] N. A. Mohammed, S. Hassan, F. Malek, R. Ngah, and S. A. Abbas, "Optical generation of 60-GHz signal for millimeter wave wireless communication," in 2013 IEEE International RF and Microwave Conference (RFM), pp. 437-440, 2013.

[21] M. Baskaran and R. Prabakaran, "Optical millimeter wave signal generation with frequency 16-tupling using cascaded MZMs and no optical filtering for radio over fiber system," Journal of the European Optical SocietyRapid Publications, vol. 14, no. 13, p. 1-8, 2018. 
[22] N. A. Al-Shareefi, et al., "Development of a new approach for high-quality quadrupling frequency optical millimeter-wave signal generation without optical filter," Progress in Electromagnetics Research, vol. 134, pp. 189-208, 2013.

[23] P. Shi, et al., "A frequency sextupling scheme for high-quality optical millimeter-wave signal generation without optical filter," Optical Fiber Technology, vol. 17, no. 3, pp. 236-241, 2011.

[24] L. Shang, et al., "A filterless optical millimeter-wave generation based on frequency octupling," Optik-International Journal for Light and Electron Optics, vol. 123, no. 13, pp. 1183-1186, 2012.

[25] J. Ma, et al., "Fiber Dispersion Influence on Transmission of the Optical Millimeter-Waves Generated Using LNMZM Intensity Modulation," in Journal of Lightwave Technology, vol. 25, no. 11, pp. 3244-3256, Nov. 2007. doi: 10.1109/JLT.2007.907794. 